\title{
Uncertainty in the manufacturing of fibrous thermosetting composites: A review
}

\author{
T.S.Mesogitis ${ }^{\mathrm{a}^{*}}$, A.A.Skordos ${ }^{\mathrm{a}}$, A.C.Long ${ }^{\mathrm{b}}$ \\ a School of Applied Sciences, Manufacturing and Materials Department, Composites Centre, \\ Cranfield University, Bedford, MK43 0AL, UK \\ ${ }^{\mathrm{b}}$ Division of Materials, Mechanics \& Structures, University of Nottingham, University Park, \\ Nottingham NG7 2RD, UK \\ tel: + 44 (0) 1234752 797; fax: + (0) 1234750875
}

*corresponding author's email: t.mesogitis@cranfield.ac.uk

Keywords: A. Thermosetting resin, C. Statistical properties/methods, C. Probabilistic methods

\begin{abstract}
Composites manufacturing involves many sources of uncertainty associated with material properties variation and boundary conditions variability. In this study, experimental and numerical results concerning the statistical characterization and the influence of inputs variability on the main steps of composites manufacturing including process-induced defects are presented and analysed. Each of the steps of composite manufacturing introduces variability to the subsequent processes, creating strong interdependencies between the process parameters and properties of the final part. The development and implementation of stochastic simulation tools is imperative to quantify process output variabilities and develop optimal process designs in composites manufacturing.
\end{abstract}

\section{Introduction}

The manufacturing process of composite materials involves many uncertainties which can result in a considerable amount of scrap associated with significant cost and environmental implications. 
Furthermore, the existence of defects generated due to variability can compromise the performance of composite components, leading to the use of more conservative designs that do not fully exploit the performance and environmental opportunities offered by composites. These uncertainties can be summarised as follows [1,2]:

(i) fibre architecture variations which are usually generated during production, handling or storage of pre-pregs, dry textiles and performs.

(ii) matrix material uncertainties caused by variations in storage conditions or uncertainties in resin composition and formulation.

(iii) variations in environmental parameters and process conditions.

Fibre heterogeneity can significantly affect the forming/draping step [3], as well as introduce permeability and thermal property uncertainty affecting the filling and curing steps of processing. Furthermore, fibre architecture governs the structural performance of components with local variability playing a critical role in non-linear phenomena such as failure and damage. Matrix material uncertainties influence the filling and curing stages which in turn influence the quality of the final product. Variations in process parameters may affect all manufacturing steps and consequently the quality of the component. A design approach that would take these effects into account explicitly would need to be based on stochastic simulations of composites manufacturing which would allow quantification of process outcome variability as a function of material selection and process parameter definition decisions made at an early stage.

Stochastic simulation involves four main steps; a) quantification of the input variable uncertainties (uncertainty quantification), b) development of a stochastic model representing the variability of uncertain parameters and their cross correlation (stochastic model), c) implementation of a model that propagates uncertainty through a deterministic process model (propagator), d) quantification of the output parameters uncertainty $[4,5]$. The input variables are considered to be either time independent random parameters which can be described by multivariate probability distributions or random fields, or time dependent stochastic processes described by stochastic differential equations. The random fields or stochastic processes are uncovered by carrying out relevant experiments. 
The aim of the present paper is to summarise the state of the art on experimental and stochastic simulation methodologies and results focusing on statistical characterisation and the influence of inputs variability on the main steps of composites manufacturing including process-induced defects as well as to highlight the interdependencies between the process parameters. Uncertainty introduced by experimental methods and modelling practices is also included.

\section{Stochastic simulation methods}

Stochastic simulation methods can be divided into two categories; intrusive and non-intrusive. Intrusive techniques involve reformulation of the main model equations while non-intrusive techniques treat the main model as an independent model. The most common non-intrusive method is the Monte Carlo scheme, which is a sampling technique used to generate random samples of input variables values from their respective statistical distributions [4]. Since random sampling is used, a quite large number of the deterministic main model runs is usually required to ensure convergence and accuracy, leading to high computational cost, especially in the case of complex and multidimensional stochastic problems [6]. The Spectral Stochastic Finite Element (SSFEM) method is the most common intrusive technique [7]. It uses the Karhunen-Loève (K-L) expansion to discretise the input random field and the polynomial chaos expansion to represent the output variables using a set of orthogonal functions [7]. The coefficients of the polynomial chaos expansion are calculated using the probabilistic Galerkin approach. The domain of the solution incorporates the probability space resulting in a system of equations significantly larger than that of the deterministic problem, with the associated increase in computational costs [6]. The Probabilistic Collocation method offers an intermediate solution between Monte Carlo and stochastic finite elements. This method is similar to the SSFEM using both the $\mathrm{K}-\mathrm{L}$ expansion and the polynomial chaos expansion to represent the input and output random fields, respectively. However, the unknown polynomial chaos coefficients are calculated by the probabilistic collocation approach, which is also a weighted technique for minimising residuals. The collocation points are the roots of the next higher order orthogonal polynomial for each stochastic parameter and are chosen so that the residuals between the polynomial chaos expansions and model outputs approach zero, implying that the collocation points are selected 
from regions of higher probability. Consequently, a system of linear equations is obtained for every output parameter. Using this sampling method, no reformulation of the deterministic model is required, which is solved several times for each collocation point. This of course has significant benefits in terms of computational efficiency when the number of stochastic components is relatively low [6].

The capabilities of the collocation method have been demonstrated in the context of composite manufacturing in the case of simulation of RTM filling. The results indicated the capability of the technique and its significant benefits compared to Monte Carlo [8]. More details concerning the SSFEM and the probabilistic collocation method can be found in [6,7].

\section{Variability of dry textiles and pre-pregs}

Variability is present in all forms of textile reinforcements including pre-pregs and dry textiles [9]. Variability in as supplied-dry reinforcements and pre-pregs is associated with tow waviness (Fig. 1), tow size and shape variations, distribution of fibres inside the tows, resin content variations and is generated during production, handling or storage [9-12]. For instance, the alignment and stiffness characteristics of the rollers used during the production of pre-pregs, can sometimes cause resin content variations, or the way pre-pregs are wrapped on to a drum for storage can cause wrinkles which in turn may result in considerable tow misalignment $[9,12]$. Geometrical variability of tows spreads to adjacent locations due to friction forces at tow crossovers (woven textiles) and fibre continuity [10] resulting in spatially correlated random fields of the uncertain variables. Fibre orientation variability can be described by a normal distribution [10,13-16] combined with strong spatial autocorrelation spread over several unit cells of the textile [10]. An experimental investigation of the internal geometry of 3D woven textiles using micro-computed tomography underlined the importance of variability in dry reinforcements [17]. The coefficient of variation of the dimensions of the tows and the inter-tow spacing reaches values of 16 and 6\%, respectively. Experimental results on the internal geometry of a non-crimp woven fabrics show variability in the range of $4-8 \%$ for the tows dimensions, of 3-4\% for tow spacing [18]. These sources of variation exist in the reinforcement in its 
as received state, setting the minimum level of uncertainty in all subsequent steps of composite manufacturing.

\section{Uncertainty in forming/draping}

The forming/draping stage of the manufacturing process causes significant shear deformation in the case of doubly curved components [19]. There are two main mechanisms of shear deformation during the forming/draping step; scissoring i.e. the change in the inter-fibre angle, and inter-fibre sliding which occurs in processes involving extremely high deformations $[20,21]$. These phenomena can lead to wrinkles and can be eliminated using localised stitching [22]. Shear deformation has significant impact on local fibre volume fraction and thickness; regions of higher deformation present an increase in fibre volume fraction or thickness depending on whether the tooling used is rigid or not [21]. Prepregs and dry textiles are not perfectly aligned therefore additional localised buckling and wrinkling may occur affecting the forming behaviour of the material [23]. Stochastic simulation shows that tow orientation uncertainties can cause significant variations in the outcome of the forming of woven composites, with coefficients of variation of minimum and average wrinkling strain in the range of $10-20 \%$ [10]. Initial tow waviness of as-received unidirectional pre-pregs may have beneficial effects during draping, since additional waviness can be introduced instead of tow misalignment, which may eliminate the formation of wrinkles [12]. Apart from the initial fibre angle variations, boundary conditions uncertainty, such as variations in the blank holder force may also influence the forming step [24]. Furthermore, the fact that there is a large number of ways to drape over a complex geometry implies additional uncertainty of the process $[9,12]$. In general the manifestation of variability is more pronounced when manual work is involved. Mechanical conditioning is considered to be a way to increase repeatability and to reduce variability since the tow tensions due to weaving can be balanced [19]. However, it is very difficult to apply mechanical conditioning in industrial applications unless the handling is adapted for this purpose.

Therefore, the shear behaviour of reinforcements can show significant variations from part to part. As it will be reported in the next sections, these effects can influence a wide variety of parameters such as the permeability, the development of residual stresses during the curing stage, dimensional accuracy, 
and the mechanical properties of the final part indicating the presence of strong interdependencies of various sources of variability in composite manufacturing [9,20-22].

\section{Uncertainty during impregnation/consolidation}

Permeability of dry reinforcement is the key parameter controlling the impregnation step in liquid composite moulding processes. Significant amount of research has been carried out concerning permeability evaluation to allow consideration of process issues such as formation of dry spots and voids; extended impregnation cycles, uneven impregnation and resin rich pockets [25]. Preform architecture variability due to different handling and storage conditions or shear deformations during the forming/draping stage, nesting effects during lay-up, low resistance channels along the preform causing macroscopic and microscopic voids, and random experimental errors result in considerable permeability variations [11,26-28]. Other sources of uncertainty in the impregnation stage can be resin viscosity variations, foreign material inclusion during impregnation, preform volume fraction variations, as well as accidental misplacement of the preform in the mould [5,29]. Experimental and simulations results using non-crimp fabrics have shown that there is a linear relationship between the coefficient of variation of permeability and the coefficient of variation of fibre volume fraction [13,30]. Several experimental and simulation studies have outlined the stochastic nature of permeability. Relative standard deviations up to $20 \%$ were observed during permeability measurements [14,26,27,31-36], while according to other results permeability relative standard deviation can reach values up to $30 \%$ [13] (Table 1). The high scatter observed in [13] is probably due to the small number of experiments compared to the other studies. Nesting is one of the primary reasons for this large scatter in permeability [27]. In addition, experimental results have indicated that the anisotropy ratio of in-plane permeability which dictates the filling pattern and thus is of crucial importance for mould design can also show strong variability following a lognormal distribution [27]. Different anisotropy distributions among different textiles are observed, even when the distribution of the principal permeability values is similar [37]. For instance, a plain weave fabric shows the largest scatter in anisotropy, while a twill weave shows the smallest. This implies that the principal permeability values may show strong correlation in some preforms, whereas in other materials little or 
no correlation may be observed [37]. Preforms with strong correlation and small anisotropy scatter, are expected to be manufactured more consistently. The flow pattern can considerably vary even for configurations of the same porosity, implying that the latter alone cannot be used to predict permeability [38]. Furthermore, for porosities in the range of $0.45-0.7$, transverse permeability decreases as the fibre heterogeneity increases [38]. This phenomenon is attributed to the formation of narrow gaps between the fibres which lead to permeability reduction. Permeability can show considerable variations at the micro-scale; inside the fibre bundles [39]. These findings indicate that permeability should be described as a stochastic variable and thus a large number of experiments are required to measure it properly [37]. According to Hoes [27], at least 20 experiments are required to have a proper estimate of the mean value, whereas at least 30 experiments are required to have a proper estimate of the standard deviation. Similarly with tow orientation, it has been shown that global permeability values can be described by a normal distribution [13-16]. However, simulation results indicated that permeability at the mesoscale (unit cell size) cannot be represented by a normal distribution [30]. This was also observed in the case of a random mat [40].

\subsection{Preform heterogeneity effects}

As mentioned in section 4, during the draping step the preform is subjected to significant shear deformation intensifying the already existing geometrical heterogeneities. As a result, the draping step affects significantly the local permeability values and thus the flow rate and the injection pressure by altering the permeability anisotropy ratio, fibre volume fraction and porosity distribution in the textile $[21,25,26]$. The permeability decreases in a non-linear manner with increasing fibre volume fraction [21,27]. Consequently, one would expect that shear deformation caused during draping would always reduce the local permeability values in the regions of high shear. However, flow visualization results indicate that in some cases high volume fraction regions caused by shear deformation during draping, may result in higher permeability values. The effect of draping on permeability is characterised by three competing effects; the reduction of local permeability due to higher fibre volume fraction in the regions of high shear, reorientation of fibres due to shear leading to reorientation of the permeability principal axes, and the fact that a smaller amount of resin is required to saturate the textile in the 
regions of high volume fraction. Therefore, although fibre volume fraction is higher at these regions, the flow front can be faster [21]. Similar results are found in radial injection experiments, in which the permeability in the fibre direction was found to increase slightly with increasing shear up to a maximum, followed by a reduction, whereas transverse permeability was found to decrease continuously as the shear angle increases [25]. Consequently, when the fibre volume fraction effects dominate, the permeability is locally reduced due to shear deformation, while the opposite effect occurs when the influence of the reorientation of the permeability principal axes is more pronounced. While preform permeability is dependent on fibre distribution at microscopic level, i.e. within fibre tows and bundles, [28] as well as at macroscopic level, i.e. fibre angle uncertainties, the flow front shape is locally determined by the size of the unit cell $[1,2,13]$. Fibre architecture variability at macroscopic level can be described by the fibre angle variation, and the fibre-void distribution $[2,13]$. Since geometrical uncertainties are spread to adjacent locations in the material, the in plane fibre spacing can be modelled by continuous random fields based on a spectral expansion [1]. Stochastic simulations based on this approach as well as experimental results, showed that although the global permeability distribution is governed by local permeability variations [29] (global permeability is the spatial harmonic mean of the local permeability), global and local permeability uncertainties should be differentiated [1,13]. Fibre-void distribution heterogeneities imply high angle variations, which consequently result in higher local permeability variations. The global permeability variations in turn increase rapidly with fibre angle variations, until they reach a maximum. Thus, there is a critical point beyond which the global permeability variations start to decrease. Nonetheless the local variations are high, at the global scale; the textile appears to be more uniform leading to lower global variances [13]. In the case of smaller moulds the effect of fibre misalignment at the mesoscale, whether large or small is dominant. On the other hand, as the mould dimensions increase, these local inhomogeneities tend to cancel each other out and the textile appears to be more uniform leading to lower global permeability variations $[13,30]$. This suggests that the global permeability distribution is governed by the mould dimensions; larger moulds lead to lower global permeability variations [30]. 


\subsection{Nesting effects}

Nesting during the lay-up process is governed by both mechanical and geometric phenomena, and it is of crucial importance as it affects the permeability, the thermal conductivity and the mechanical behavior of the composite. In general, nesting affects the laminate thickness, the fibre volume fraction and the pore pattern. Therefore nesting can introduce significant spatial scatter in laminate properties at different locations over a composite part, as well as considerable batch to batch variability [30-34]. Compressibility studies have clearly indicated that irrespective of fabric type, for a given pressure, the thickness per layer decreases due to nesting [30-35]. However, in the results presented in [36], the thickness per layer increased with increasing the number of lamina layers, as a result of friction between the layers which prevented the occurrence nesting.

Several experimental and computational studies have identified the importance of nesting on permeability variation, implying that nesting variability is the main reason for permeability scatter. Simulations investigating the effect of nesting on the permeability of plain weave fabrics showed that permeability values can vary by an order of magnitude between maximum and zero nesting conditions [37,38]. Experimental results using a large number of samples demonstrate a high permeability variation; the highest measured value can be three times the lowest one [15]. The same order of scatter can be identified in local permeability calculations using the pore network technique [39].

Nesting can show considerable scatter associated with several parameters. It has been shown that shear deformation has a great impact on nesting. In particular, a textile is less prone to nesting if it is subjected to shear deformation [40]. Consequently, at higher shear angles the scatter in thickness per layer is reduced.

Furthermore, nesting can be confined by increasing the tightness of the textiles. When the fibre tows are placed tighter, the "hills and valleys" of fibre tows are steeper and nesting is more difficult to occur. Inter-tow spacing also affects nesting. The textile tightness decreases as the inter-tow spacing increases, and hence nesting is less pronounced leading to lower thickness variation. Therefore, tow angle variations influence indirectly nesting, as they are associated with inter-tow spacing. In the case of non-crimp fabrics the stitching pattern dictates nesting [40]. Nesting is also influenced by the tool 
properties, such as mould stiffness and surface condition, as well as the compression force during consolidation. These effects have not been investigated so far.

Another phenomenon associated with compressibility and nesting effects is the formation of resin rich zones. Resin rich zones are usually formed in the gaps between the internal mould surface and the textile preform, as well as in the fabric, around stiches [41]. During consolidation shifting of the preform may also result in resin rich pockets [9]. In the case of curved parts resin rich zones may be formed due to the fact that the preform tends to fit tightly around the corner regions [41]. Furthermore, nesting of individual fibre tows may cause local resin rich zones in the regions between the tows. As a result, resin rich zones can lead to additional out of plane tow misalignment having detrimental effects on the mechanical properties of the final part [9]. The formation of resin rich pockets is difficult to predict, and consequently introduces considerable variability in the process. The magnitude of the gap between the mould inner surface and the preform is a function of the friction between the mould and the preform as well as of the compressibility of the preform [41]. This implies that uncertainties on tooling properties such as friction coefficient due to repetitive usage or variations on the properties and the amount of release agent may influence the formation of resin rich pockets.

\subsection{Edge effects}

Race tracking is a source of preform permeability uncertainty during liquid moulding processes [42]. Race tracking is inevitable in industrial applications and refers to edge effects caused by imperfect fit between the preform and the edges of the mould [43-45]. As a result, low flow resistance channels are formed along the edges, where the resin flow front moves faster and the local permeability is considerably higher $[43,44]$. This phenomenon introduces significant variability since it is only dependent on how the textile is cut and placed into the mould cavity [44]. A statistical study showed that the permeability values caused by race-tracking can be described by a Weibull distribution [43]. Therefore, in the case of one-dimensional permeability experiments where race tracking is likely, the Weibull distribution can be used to characterize permeability instead of the conventional normal distribution [46]. 


\subsection{Void formation}

Liquid composite moulding processes often suffer from flow-induced voids leading to poor part quality. Entrapped voids can influence the stress distribution in the part and lead to stress concentrations, which can eventually result in local failure. Experimental studies have shown that for a $1 \%$ increase in void content, the flexural strength, flexural modulus, and inter-laminar shear strength can decrease more than 5\% [47-49]. Void formation also reduces the fatigue strength and durability of the material and makes it more susceptible to environmental conditioning and moisture absorption. Due to these detrimental effects, the void content should be minimised as much as possible. The mechanisms leading to entrapped voids are associated with preferential flow channels arising from preform heterogeneities which in turn lead to permeability variations [50]. Thus, void formation can be considered to be a directional phenomenon [51]. However, in some cases void formation is not related to resin flow, and can be attributed to initial resin air content, release agent evaporation and volatiles formed by the reaction products during cure [50]. These mechanisms are more pronounced in autoclave processes [52].

Two distinct flow patterns occur during resin impregnation due to fibre architecture heterogeneities: viscous flow, which is pressure driven and dominates in macro-pores and capillary flow which is dominant in micro-pores due to capillary forces $[52,53]$. The flow front is usually uneven between these two mechanisms, leading to the formation of voids. The viscous flow leads the capillary flow at high injection rates, resulting in microscopic intra-tow voids caused by fingering, whereas, at low injection rates, the capillary flow is dominant causing macroscopic inter-tow voids [53]. These phenomena are usually described by the capillary number expressed as the ratio between viscous and capillary forces [50]. The size and shape of intra-tow micro-voids are strongly affected by geometric heterogeneities inside the fibre tows as well as non-structural stitches [43]. Race-tracking can sometimes lead to macro-voids generated when flow patterns reach the vent locations prior to full saturation [44].

Image analysis of glass/epoxy composites with different volume fractions highlighted the importance of fibre volume fraction on void formation in resin transfer moulding processes [53]. Higher fibre 
volume fractions result in lower void content and void areal density. This behaviour is attributed to higher injection pressures, more uniform fibre architecture as well as lower matrix volume fraction. Three distinct types of voids can be identified; voids in resin rich areas, intra-tow voids, and inter-tow voids. Increasing the fibre volume fraction decreases the contribution of resin voids to total void content and total void areal density. On the contrary, the contribution of intra-tow voids to total void areal density increases by increasing fibre volume fraction, while the contribution to total void content is reduced, implying that the size of intra-tow voids decreases at higher volume fractions. The contribution of voids located next to fibre bundles does not show any clear dependence on fibre volume fraction. Fibre volume fraction also affects the distribution of voids along the radius of curved parts of components [53].

The size and location of voids can vary significantly. In general, voids can be classified to spherical and bigger random-shaped voids [53]. Randomly shaped voids have more severe effects on mechanical performance since they can cause premature crack initiation. The content and the aerial density of random voids decrease at higher volume fractions [53]. Consequently, the probability of premature crack initiation is reduced by increasing the fibre volume fraction. The spatial void distribution is also an important parameter since it dictates the overall performance of the final part [50]. An uneven void content distribution can arise from inter-layer space variations or space variations between the preform and the mould [50]. Moreover, the fibre content also influences spatial void distribution. At higher fibre volume fractions, formation mechanisms can be affected since the elevated injection pressure can cause considerable void shrinkage and void transportation may be facilitated towards the exit vent locations [53]. Thus, at high fibre volume fractions both preform heterogeneity and void mobility mechanisms affect spatial void distribution.

Nesting effects play an important role on void formation [16,54]. Both the magnitude and variability of dry spot content are strongly influenced by nesting and the distribution layer permeability in the case of vacuum assisted resin transfer moulding processes. Increasing the preform thickness enhances dry spot formation and its variability within the textile [54]. High scatter in by-pass paths permeability and high values of distribution layer permeability result in high scatter in dry spot content. This high scatter can be reduced by lowering the distribution layer permeability. High dry spot content variation 
implies that the formation of dry spots depends on the spatial distribution of the by-pass paths permeability.

\section{Uncertainty in composites cure}

The cure process is a complex thermo-mechanical phenomenon involving several sources of uncertainty such as material and tooling characteristics variation as well as environmental/boundary condition uncertainties. These uncertainties can affect the formation of residual stresses and can result in under-curing, over-curing, cure-induced voids and severe temperature overshoots which may cause thermal degradation $[4,9,55]$.

\subsection{Material properties and boundary conditions variation effects on cure}

The resin cure kinetics is of crucial importance having a great impact on the curing process. The parameters of cure kinetics models are usually estimated using differential scanning calorimetry (DSC), and can vary due to different handling and storage conditions of the resin or the presence of fibre sizing [56]. Particularly, handling and shelf life history uncertainties can cause variability in resin state and the initial degree of cure $[5,9,56,57]$. The thermal, mechanical, and thermo-mechanical properties of the material can show significant uncertainties affecting the cure process. These uncertainties are generated by the inherent uncertainties of the constituents as well as the previous steps of the manufacturing process. Thermal conductivity variations are caused by variations in fibre orientation and fibre volume fraction [58]. Variations in heat capacity can be caused by scatter in the heat capacity of the constituents and fibre volume fraction [58]. Thermal expansion coefficients are affected by uncertainty in the moduli and thermal expansion coefficients of the constituents, volume fraction variations and ply misalignment $[58,59]$. Fibre volume fraction variations also affect the total heat of reaction whereas mechanical properties uncertainty is a function of material properties and fibre volume fraction variations [58].

Environmental/boundary condition uncertainties including ambient temperature, moisture, convective heat, prescribed pressure and temperature can show variations introducing additional variability to the process [58,60]. Apart from environmental conditions, the convective heat is affected by the 
geometric characteristics of the tools [55] and by other random factors such as wrinkles in vacuum bags. Variability in convective heat can result in uneven curing and temperature distribution which can potentially lead to severe temperature overshoots, or even incomplete cure. The occurrence of temperature overshoots is also influenced by tool properties [55,61], implying that in the case of composite tooling, additional variability may be added.

Cure temperature and resin kinetics variations have a great impact on cure time distribution [4]. In particular, cure temperature variations dominate over resin kinetics uncertainties having the greatest influence on cure time variability, while faster reacting resins show higher cure time variability than systems with higher activation energy [4]. Unlike fibre orientation uncertainties, the variation of cure process parameters has been not explicitly quantified.

\subsection{Residual stresses-shape distortion}

The cure of composites always results in residual stresses which can lead to delamination, crack initiations, and shape distortions such as spring-in or warpage $[62,63]$. The formation of residual stresses is mainly dependent on the mechanical and thermo-mechanical properties of the constituents [41,64]. As mentioned in section 6.1, these properties are strongly influenced by the fibre volume fraction, implying that fibre volume fraction variations can introduce considerable scatter to the formation of residual stresses [41,65]. Regions with locally lower fibre volume fraction are more prone to geometrical distortions and defects due to process-induced stresses [9,65]. A variation in the order of $6 \%$ in fibre volume fraction can lead to 5\% variation in the spring-in angle [65]. Such deviations can cause serious assembly issues. The development of residual stresses is also affected by cure kinetics variations, with variability in activation energy having the greatest impact [64]. Tooling properties affect the development of residual stresses as well [66]. Numerical simulations [66] have indicated that different mould materials have different effects on the development of process-induced residual stresses and strains, implying that in the case of composite tools further uncertainties may be added. 


\section{Property measurement and model uncertainties}

There are several techniques and modelling practices to characterise the behaviour of composites during the different stages of manufacturing. The shear behaviour of preforms can be characterised using optical methods, picture frame experiments or bias-extension experiments. Sample size is of crucial importance; a larger shearing force is required with increasing sample area. Considerable discrepancies can be observed between the three methods, especially at angles above 30 degrees [19]. A round-robin study involving picture frame experiments and bias-extension experiments for different textiles, indicated deviations between the different laboratories. In both cases, the scatter increased with shear angle, being more pronounced above 30 degrees [19].

In plane permeability can be measured by two principal ways: the radial flow technique and the linear flow technique. Both of these methods have several variants such as permeability measurements during saturated or unsaturated flow as well as constant pressure or constant flow rate measurements. Since there are no standard guidelines established to date, it is debatable which technique is the most accurate [81]. It has been shown that unsaturated linear flow experiments show the highest reproducibility [31,82,83]. According to [81,84,85], linear flow experiments are consistent with radial flow experiments, with the latter showing higher variations, whilst in [86] differences were found between the unsaturated linear technique and the wetting radial technique. In the case of linear flow experiments, errors can be introduced if the flow channel axis does not coincide with the principal axis of the fabric [81]. In addition, linear experiments can be significantly influenced by race tracking [87]. As Wang et al. [88] recommended evaluation of both radial and linear flow experiments should be carried out in order to obtain accurate experimental results. Significant deviations can be observed between the saturated and unsaturated flow technique due to transient capillary effects during wetting flow experiments $[31,83,84,89]$. In general, saturated flow experiments lead to higher permeability values than those obtained from unsaturated flow experiments [81]. The different types of fluids used to measure permeability can also contribute to variability [90]. The shape of the mould can also introduce scatter in permeability measurements, implying that measurement performed using conventional geometries may not be appropriate for designing complex parts [14]. Although the 
question of sample size is still open [40], as discussed in section 5.1, in general, larger moulds lead to lower global permeability variations [30]. A scatter of the same order as the experimental error was observed during a round-robin exercise between three different laboratories. The observed scatter was related to variation in specimen preparation [31]. However, round-robin results involving 16 different experimental procedures indicated a scatter of up to one order of magnitude in principal permeability values, whereas the ratio of principal permeability varied by a factor of up to 2 . The main source of uncertainty between the different procedures was attributed to human factors including misconceptions about the experimental process, the use of unsuitable data treatment, different permeability definitions, inconsistent use of units and different specimen preparation conditions [81]. There are several cure monitoring techniques such as DSC, dielectrometry, infrared spectroscopy, and dynamic mechanical analysis (DMA). In general cure state measurements using DSC and dielectrics agree well [91], whereas significant differences are observed in the measurement of glass transition temperature between different techniques such as DSC and DMA [92]. Although DSC is the most common method to characterise cure kinetics, considerable discrepancies can be observed due to variability in baseline decision, different range of measured data, measurement quality variation, and variation in data reduction decisions. [67]. In addition, significant scatter can be observed between different cure kinetics models due to different modelling practices and the aforementioned characterisation uncertainties [67].

\section{Concluding remarks}

The fabrication of thermosetting composites is a very complex procedure, involving processes of different physics and scales. Each of the steps of composite manufacturing (forming, consolidation/impregnation and curing) introduces variability to the subsequent manufacturing processes, creating strong interdependencies between the process parameters and their variability and properties of the product. Therefore, deterministic process simulation models are not able to capture fully the real phenomena, rendering the development and implementation of stochastic simulation tools for composite manufacturing imperative. 
Fibre volume fraction seems to play the dominant role in composites manufacturing. Fibre volume fraction variations due to geometrical heterogeneities caused during the production of pre-pregs/dry textiles and forming /draping step along with nesting and edge effects can introduce significant scatter in permeability during the impregnation/consolidation step. This can result in flow-induced voids and resin rich zones further affecting the fibre volume fraction distribution. Fibre volume fraction also affects the thermal, mechanical and thermo-mechanical properties of the constituents introducing variability to the cure of composites. Furthermore, the cure process is significantly influenced by environmental/boundary condition uncertainties as well as resin property uncertainties due to different handling and storage conditions. These effects can introduce variability in residual stresses/shape distortion and can lead to serious cure induced defects. A schematic representation of these interdependencies is depicted in Fig. 2.

Unlike permeability variations, limited data exist regarding uncertainty in forming/draping. In addition, variability in composites cure has not been explicitly characterised; the results presented in the literature were obtained using conceptual values for the input variable uncertainties rather than experimental data. Therefore further investigation should be carried out towards the statistical characterization and incorporation of all the sources of uncertainty during the forming/draping and cure step.

The findings presented in this study highlight the importance of variability in composites manufacturing and thus the need for future development and incorporation of stochastic simulation schemes into the existing commercial simulation tools. This implies that stochastic simulation should play a major role in process design; adopting stochastic simulation tools will have tremendous benefits in terms of costs. Benchmark guidelines should be developed regarding characterisation techniques and modelling practices in all manufacturing steps, to minimise property measurement and model uncertainties.

\section{Acknowledgements}

This work was supported by the Engineering and Physical Sciences Research Council [grant number: EP/IO33513/1], through the EPSRC Centre for Innovative Manufacturing in Composites (CIMComp). 


\section{References}

[1] Endruweit A, Long AC. Influence of stochastic variations in the fibre spacing on the permeability of bi-directional textile fabrics. Composites Part A: Applied Science and Manufacturing 2006;37(5):679-694.

[2] Endruweit A, Long AC, Robitaille F, Rudd CD. Influence of stochastic fibre angle variations on the permeability of bi-directional textile fabrics. Composites Part A: Applied Science and Manufacturing 2006;37(1):122-132.

[3] Long A, Wiggers J, Harrison P. Modelling the Effects of Blank-Holder Pressure and Material Variability on Forming of Textile Preforms. In: Proceedings of the 8th International ESAFORM Conference on Materials Forming, Cluj-Napoca, Romania 2004.

[4] Padmanabhan SK, Pitchumani R. Stochastic analysis of isothermal cure of resin systems. Polymer Composites 1999;20(1):72-85.

[5] Padmanabhan SK, Pitchumani R. Stochastic modeling of nonisothermal flow during resin transfer molding. Int J Heat Mass Transfer 1999;42(16):3057-3070.

[6] Huang S, Mahadevan S, Rebba R. Collocation-based stochastic finite element analysis for random field problems. Prob Eng Mech 2007;22(2):194-205.

[7] Ghanem RG, Spanos PD. Stochastic finite elements: a spectral approach: Dover Pubns, 2003.

[8] Zhang F, Cosson B, Comas-Cardona S, Binetruy C. Efficient stochastic simulation approach for RTM process with random fibrous permeability. Composites Sci Technol 2011;71(12):1478-1485.

[9] Potter K, Understanding the origin of defects and variability in composites manufacture. In: Proceedings of the 17th International Conference on Composite Materials, Edinburgh, UK, 2009.

[10] Skordos AA, Sutcliffe MPF. Stochastic simulation of woven composites forming. Composites Sci Technol 2008;68(1):283-296.

[11] Verleye B, Lomov SV, Long A, Verpoest I, Roose D. Permeability prediction for the mesomacro coupling in the simulation of the impregnation stage of Resin Transfer Moulding. Composites Part A: Applied Science and Manufacturing 2010;41(1):29-35.

[12] Potter K, Khan B, Wisnom M, Bell T, Stevens J. Variability, fibre waviness and misalignment in the determination of the properties of composite materials and structures. Composites Part A: Applied Science and Manufacturing 2008;39(9):1343-1354.

[13] Endruweit A, McGregor P, Long AC, Johnson MS. Influence of the fabric architecture on the variations in experimentally determined in-plane permeability values. Composites Sci Technol 2006;66(11-12):1778-1792.

[14] Pan R, Liang Z, Zhang C, Wang B. Statistical characterization of fiber permeability for composite manufacturing. Polymer composites 2000;21(6):996-1006.

[15] Hoes K, Dinesku D, Vanhuele M, Sol H, Parnas R, Belov E, et al. Statistical distribution of permeability values of different porous materials. In: Proceedings of the 10th European conference on composite materials, Brugge; 2002.

[16] Endruweit A, Long A, Robitaille F, Rudd C. Dependence of permeability variations on the textile structure. Proceedings of ECCM, 2004. 
[17] Desplentere F, Lomov SV, Woerdeman DL, Verpoest I, Wevers M, Bogdanovich A. Micro-CT characterization of variability in 3D textile architecture. Composites Sci Technol 2005;65(13):19201930.

[18] Karahan M, Lomov SV, Bogdanovich AE, Mungalov D, Verpoest I. Internal geometry evaluation of non-crimp 3D orthogonal woven carbon fabric composite. Composites Part A: Applied Science and Manufacturing 2010;41(9):1301-1311.

[19] Cao J, Akkerman R, Boisse P, Chen J, Cheng HS, de Graaf EF, et al. Characterization of mechanical behavior of woven fabrics: Experimental methods and benchmark results. Composites Part A: Applied Science and Manufacturing 2008;39(6):1037-1053.

[20] Sidhu RMJS, Averill RC, Riaz M, Pourboghrat F. Finite element analysis of textile composite preform stamping. Composite Structures 2001;52(3-4):483-497.

[21] Bickerton S, Šimáček P, Guglielmi SE, Advani SG. Investigation of draping and its effects on the mold filling process during manufacturing of a compound curved composite part. Composites Part A: Applied Science and Manufacturing 1997;28(9-10):801-816.

[22] Duhovic M, Mitschang P, Bhattacharyya D. Modelling approach for the prediction of stitch influence during woven fabric draping. Composites Part A: Applied Science and Manufacturing 2011;42(8):968-978.

[23] Yu W, Harrison P, Long A. Finite Element Forming Simulation of NCF Considering Natural Variability of Fiber Direction. In: Proceedings of the 8th International ESAFORM Conference on Materials Forming, Cluj-Napoca, Romania, 2005.

[24] Skordos AA, Monroy Aceves C, Sutcliffe MPF. A simplified rate dependent model of forming and wrinkling of pre-impregnated woven composites. Composites Part A: Applied Science and Manufacturing 2007;38(5):1318-1330.

[25] Belov EB, Lomov SV, Verpoest I, Peters T, Roose D, Parnas RS, et al. Modelling of permeability of textile reinforcements: lattice Boltzmann method. Composites Sci Technol 2004;64(7-8):1069-1080.

[26] Hoes K, Dinescu D, Sol H, Vanheule M, Parnas RS, Luo Y, et al. New set-up for measurement of permeability properties of fibrous reinforcements for RTM. Composites Part A: Applied Science and Manufacturing 2002;33(7):959-969.

[27] Hoes K, Dinescu D, Sol H, Parnas RS, Lomov S. Study of nesting induced scatter of permeability values in layered reinforcement fabrics. Composites Part A: Applied Science and Manufacturing 2004;35(12):1407-1418.

[28] Ding L, Shih C, Liang Z, Zhang C, Wang B. In situ measurement and monitoring of whole-field permeability profile of fiber preform for liquid composite molding processes. Composites Part A: Applied Science and Manufacturing 2003;34(8):779-789.

[29] Opperer JG, Kim SK, Daniel IM. Characterization of local preform defects in resin transfer molding by the gas flow method and statistical analysis. Composites Sci Technol 2004;64(1314):1921-1935.

[30] Wong C, Long A. Modelling variation of textile fabric permeability at mesoscopic scale. Plastics, rubber and composites 2006;35(3):101-111.

[31] Lundström TS, Stenberg R, Bergström R, Partanen H, Birkeland PA. In-plane permeability measurements: a nordic round-robin study. Composites Part A: Applied Science and Manufacturing 2000;31(1):29-43. 
[32] Luthy T, Hintermann M, Mosler H, Ziegmann G, Ermanni P. Dependence of the 1-D permeability of fibrous media on the fiber volume content: comparison between measurement and simulation. Structures and Materials 1998;3:433-442.

[33] Steenkamer DA, McKnight S, Wilkins D, Karbhari V. Experimental characterization of permeability and fibre wetting for liquid moulding. J Mater Sci 1995;30(12):3207-3215.

[34] Chick JP, Rudd C, Van Leeuwen P, Frenay T. Material characterization for flow modeling in structural reaction injection molding. Polymer composites 1996;17(1):124-135.

[35] Hammond V, Loos A. The effects of fluid type and viscosity on the steady-state and advancing front permeability behavior of textile preforms. J Reinf Plast Compos 1997;16(1):50-72.

[36] Dunkers JP, Phelan FR, Zimba CG, Flynn KM, Sanders DP, Peterson RC, et al. The prediction of permeability for an epoxy/E-glass composite using optical coherence tomographic images. Polymer composites 2001;22(6):803-814.

[37] Liu Q, Parnas RS, Giffard HS. New set-up for in-plane permeability measurement. Composites Part A: Applied Science and Manufacturing 2007;38(3):954-962.

[38] Chen X, Papathanasiou TD. The transverse permeability of disordered fiber arrays: a statistical correlation in terms of the mean nearest interfiber spacing. Transp Porous Media 2008;71(2):233-251.

[39] Bechtold G, Ye L. Influence of fibre distribution on the transverse flow permeability in fibre bundles. Composites Sci Technol 2003;63(14):2069-2079.

[40] Zhang F, Comas-Cardona S, Binetruy C. Statistical modeling of in-plane permeability of nonwoven random fibrous reinforcement. Composites Science and Technology 2012;72(12):1368-1379.

[41] Endruweit A, Ermanni P. The in-plane permeability of sheared textiles. Experimental observations and a predictive conversion model. Composites Part A: Applied Science and Manufacturing 2004;35(4):439-451.

[42] Loix F, Badel P, Orgéas L, Geindreau C, Boisse P. Woven fabric permeability: From textile deformation to fluid flow mesoscale simulations. Composites Sci Technol 2008;68(7-8):1624-1630.

[43] De Parseval Y, Roy RV, Advani S. Effect of local variations of preform permeability on the average permeability during resin transfer molding of composites. ANTEC'95. 1995;2:3040-3044.

[44] Chen B, Lang EJ, Chou T. Experimental and theoretical studies of fabric compaction behavior in resin transfer molding. Materials Science and Engineering: A 2001;317(1-2):188-196.

[45] Chen B, Chou T. Compaction of woven-fabric preforms: nesting and multi-layer deformation. Composites Sci Technol 2000;60(12-13):2223-2231.

[46] Saunders R, Lekakou C, Bader M. Compression and microstructure of fibre plain woven cloths in the processing of polymer composites. Composites Part A: Applied Science and Manufacturing 1998;29(4):443-454.

[47] Saunders RA, Lekakou C, Bader MG. Compression in the processing of polymer composites 1. A mechanical and microstructural study for different glass fabrics and resins. Composites Sci Technol 1999;59(7):983-993.

[48] Lomov SV, Verpoest I. Compression of woven reinforcements: a mathematical model. J Reinf Plast Compos 2000;19(16):1329-1350.

[49] Luo Y, Verpoest I. Compressibility and relaxation of a new sandwich textile preform for liquid composite molding. Polymer composites 1999;20(2):179-191. 
[50] Pearce N, Summerscales J. The compressibility of a reinforcement fabric. Composites Manuf 1995;6(1):15-21.

[51] Dungan F, Senoguz M, Sastry A, Faillaci D. Simulations and Experiments on Low-Pressure Permeation of Fabrics: Part I-3D Modeling of Unbalanced Fabric. J Composite Mater 2001;35(14):1250-1284.

[52] Senoguz M, Dungan F, Sastry A, Klamo J. Simulations and Experiments on Low-Pressure Permeation of Fabrics: Part II-The Variable Gap Model and Prediction of Permeability. J Composite Mater 2001;35(14):1285-1322.

[53] Delerue JF, Lomov SV, Parnas R, Verpoest I, Wevers M. Pore network modeling of permeability for textile reinforcements. Polymer composites 2003;24(3):344-357.

[54] Lomov SV, Verpoest I, Peeters T, Roose D, Zako M. Nesting in textile laminates: geometrical modelling of the laminate. Composites Sci Technol 2003;63(7):993-1007.

[55] Dong C. Model development for the formation of resin-rich zones in composites processing. Composites Part A: Applied Science and Manufacturing 2011;42(4):419-424.

[56] Parnas RS, Flynn KM, Dal-Favero ME. A permeability database for composites manufacturing. Polymer Composites 1997;18(5):623-633.

[57] Li J, Zhang C, Liang R, Wang B. Statistical characterization and robust design of RTM processes. Composites Part A: Applied Science and Manufacturing 2005;36(5):564-580.

[58] Lawrence JM, Fried P, Advani SG. Automated manufacturing environment to address bulk permeability variations and race tracking in resin transfer molding by redirecting flow with auxiliary gates. Composites Part A: Applied Science and Manufacturing 2005;36(8):1128-1141.

[59] Lawrence JM, Barr J, Karmakar R, Advani SG. Characterization of preform permeability in the presence of race tracking. Composites Part A: Applied Science and Manufacturing 2004;35(12):13931405 .

[60] Li J, Zhang C, Liang Z, Wang B. Stochastic simulation based approach for statistical analysis and characterization of composites manufacturing processes. J Manuf Syst 2006;25(2):108-121.

[61] Judd NCW, Wright W. Voids and their effects on the mechanical properties of composites- an appraisal. SAMPE J 1978;14:10-14.

[62] Ghiorse S. Effect of void content on the mechanical properties of carbon/epoxy laminates. SAMPE quarterly 1993;24(2):54-59.

[63] Bowles KJ, Frimpong S. Void effects on the interlaminar shear strength of unidirectional graphite-fiber-reinforced composites. J Composite Mater 1992;26(10):1487-1509.

[64] Hamidi YK, Aktas L, Altan MC. Formation of microscopic voids in resin transfer molded composites. Journal of engineering materials and technology 2004;126:420.

[65] Avila AF, Morais DTS. A multiscale investigation based on variance analysis for hand lay-up composite manufacturing. Composites Sci Technol 2005;65(6):827-838.

[66] Park CH, Lebel A, Saouab A, Bréard J, Lee WI. Modeling and simulation of voids and saturation in liquid composite molding processes. Composites Part A: Applied Science and Manufacturing 2011;42(6):658-668. 
[67] Hamidi YK, Dharmavaram S, Aktas L, Altan MC. Effect of Fiber Content on Void Morphology in Resin Transfer Molded E-Glass/Epoxy Composites. Journal of Engineering Materials and Technology 2009;131:021014.

[68] Markicevic B, Heider D, Advani SG, Walsh S. Stochastic modeling of preform heterogeneity to address dry spots formation in the VARTM Process. Composites Part A: Applied Science and Manufacturing 2005;36(6):851-858.

[69] Guo Z, Du S, Zhang B. Temperature field of thick thermoset composite laminates during cure process. Composites Sci Technol 2005;65(3-4):517-523.

[70] Hsiao K, Little R, Restrepo O, Minaie B. A study of direct cure kinetics characterization during liquid composite molding. Composites Part A: Applied Science and Manufacturing 2006;37(6):925933.

[71] Kessler MR, White SR. Cure kinetics of the ring-opening metathesis polymerization of dicyclopentadiene. Journal of Polymer Science Part A: Polymer Chemistry 2002;40(14):2373-2383.

[72] Liaw D, Singhal S, Murthy P, Chamis CC. Quantification of uncertainties in composites. In: Proceedings of the 34th AIAA/ASME/ASCE/AHS/ASC Structures, Structural Dynamics and Materials Conference, La Jolla, CA, USA, 1993, p. 1163-1173.

[73] Chamis CC. Probabilistic composite mechanics assurance for better-cheaper-faster products. In: Proceedings of the 39th AIAA/ASME/ASCE/AHS/ASC Structures, Structural Dynamics, and Materials Conference and Exhibit, Long Beach, CA, USA, 1998, p. 1940-1951.

[74] Elseifi M. Probabilistic analysis of thick composite plates with manufacturing and material uncertainties. In: Proceedings of the 42nd AIAA/ASME/ASCE/AHS/ASC Structures, Structural Dynamics, and Materials Conference and Exhigbit, Seattle, WA, USA, 2001.

[75] Telikicherla MK, Altan MC, Lai FC. Autoclave curing of thermosetting composites: Process modeling for the cure assembly. Int Commun Heat Mass Transfer 1994;21(6):785-797.

[76] Li H, Foschi R, Vaziri R, Fernlund G, Poursartip A. Probability-based modelling of composites manufacturing and its application to optimal process design. J Composite Mater 2002;36(16):19671991.

[77] Hilton HH, Yi S. Stochastic delamination simulations of nonlinear viscoelastic composites during cure. Journal of Sandwich Structures and Materials 1999;1(2):111-127.

[78] Hsiao KT. Uncertainty modeling of residual stress development in polymeric composites manufactured with resin transfer molding process. In: Proceedings of ASME International Mechanical Engineering Congress and Exposition, Seattle, Washington, USA, 2007.

[79] Hubert P, Pipes RB, Grimsley BW. Variability analysis in vacuum assisted resin transfer molding. In: Proceedings of the 23rd SAMPE Europe International Conference, Port de Versailles, France, 2002.

[80] Antonucci V, Cusano A, Giordano M, Nasser J, Nicolais L. Cure-induced residual strain build-up in a thermoset resin. Composites Part A: Applied Science and Manufacturing 2006;37(4):592-601.

[81] Arbter R, Beraud J, Binetruy C, Bizet L, Bréard J, Comas-Cardona S, et al. Experimental determination of the permeability of textiles: a benchmark exercise, Composites Part A: Applied Science and Manufacturing 2011;42(9):1157-1168.

[82] Parnas RS, Salem AJ. A comparison of the unidirectional and radial in-plane flow of fluids through woven composite reinforcements, Polymer Composites 1993;14,(5): 383-394. 
[83] Gebart BR, Lidström P. Measurement of in-plane permeability of anisotropic fiber reinforcements. Polymer Composites 1996;17(1):43-51.

[84] Parnas RS, Howard JG, Luce TL, Advani SG. Permeability characterization. Part 1: A proposed standard reference fabric for permeability. Polymer Composites 1995;16(6):429-445.

[85] Ahn SH, Lee WI, Springer GS. Measurement of the three-dimensional permeability of fiber preforms using embedded fiber optic sensors. J Composite Mater 1995;29(6):714-733.

[86] Lekakou C, Johari MAK, Norman D, Bader MG. Measurement techniques and effects on inplane permeability of woven cloths in resin transfer moulding. Composites Part A: Applied Science and Manufacturing 1996;27(5):401-408.

[87] Bickerton S, Advani S, Mohan R, Shires D. Experimental analysis and numerical modeling of flow channel effects in resin transfer molding. Polymer composites 2000;21(1):134-153.

[88] Wang T, Wu C, Lee L. In-plane permeability measurement and analysis in liquid composite molding. Polymer composites 1994;15(4):278-288.

[89] Pillai KM. Modeling the unsaturated flow in liquid composite molding processes: a review and some thoughts. Journal of Composite Materials 2004;38(23): 2097-2118.

[90] Dykeman D. Minimizing uncertainty in cure modeling for composites manufacturing. $\mathrm{PhD}$ Thesis, The University of British Columbia, Vancouver, 2008.

[91] Bang KG, Kwon JW, Lee DG, Lee JW. Measurement of the degree of cure of glass fiber-epoxy composites using dielectrometry. Journal of Materials Processing Technology 2001;113(1):209-214.

[92] Mulligan D, Gnaniah S, Sims G. Thermal analysis techniques for composites and adhesives Second Edition, National Physical Laboratory, 2000. 


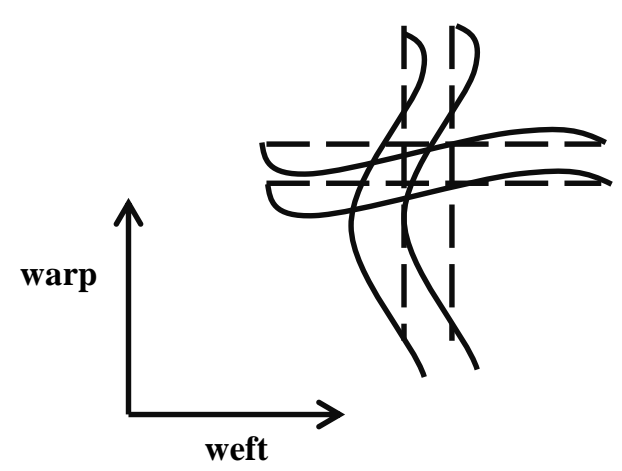

Fig. 1 Tow waviness

\begin{tabular}{|c|c|c|c|c|c|}
\hline Material & Dimensions & $\mathrm{V}_{\mathrm{f}}$ & $N_{\exp }$ & $K_{1}\left(10^{-10} \mathrm{~m}^{2}\right)$ & $K_{2}\left(10^{-10} \mathrm{~m}^{2}\right)$ \\
\hline Plain weave glass fibre 1 & $200 \mathrm{~mm}$ (circular) & $53 \%$ & 19 & $1.24 \pm 0.36( \pm 29.2 \%)$ & $0.65 \pm 0.167( \pm 25.7 \%)$ \\
\hline Plain weave glass fibre 2 & $300 \times 300 \mathrm{~mm}$ & $41.7 \%$ & 85 & $1.79 \pm 0.4( \pm 22.3 \%)$ & $1.44 \pm 0.29( \pm 20 \%)$ \\
\hline
\end{tabular}

Table 1 Permeability measurements for two plain weave glass fibre fabrics; plain weave glass 1 [13], plain weave glass fabric 2 [27] 


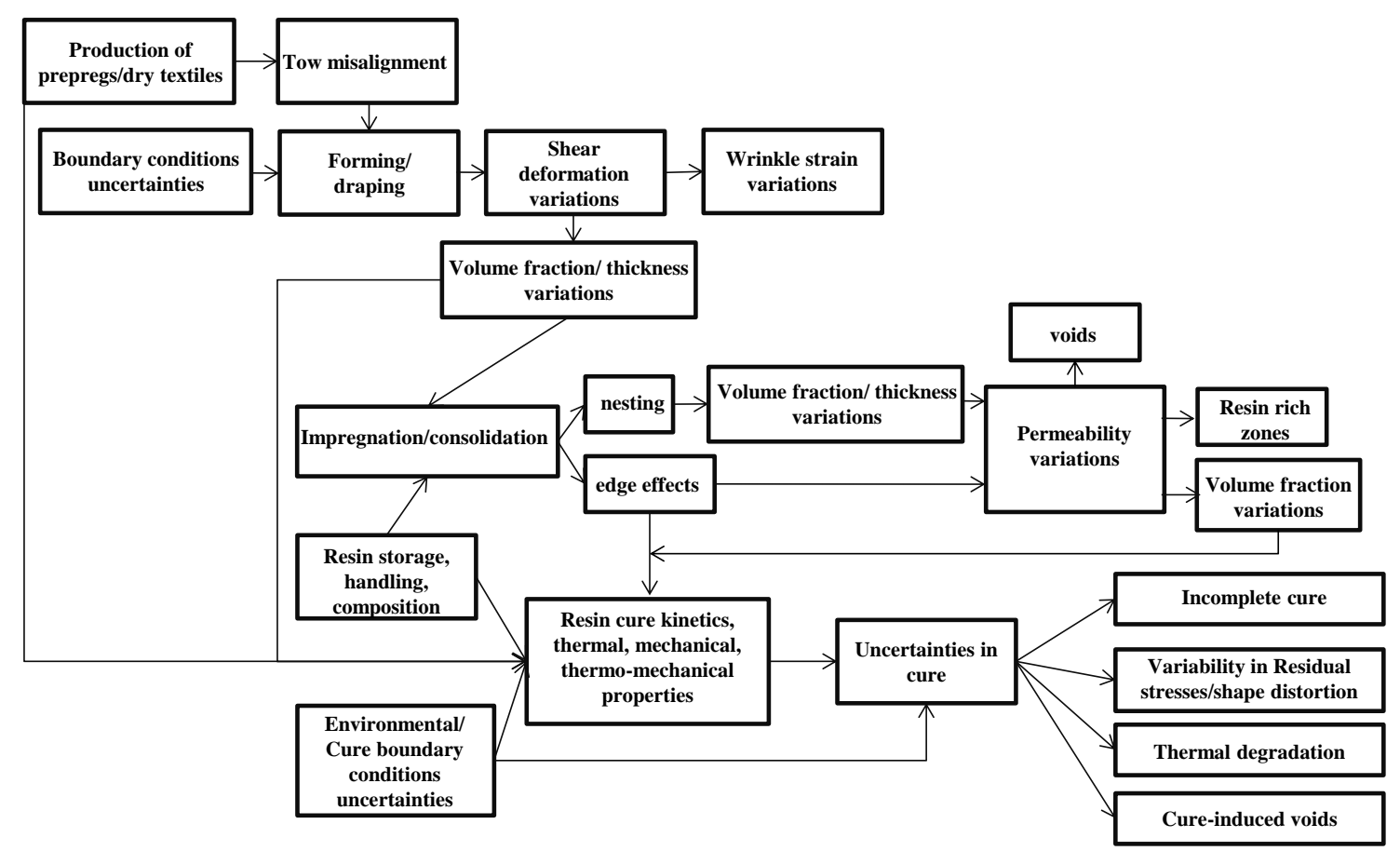

Fig. 2 Schematic representation of interdependencies in composite manufacturing 
2014-02

\section{Uncertainty in the manufacturing of \\ fibrous thermosetting composites: A review}

Mesogitis, Tassos

Elsevier

T.S.Mesogitis, A.A.Skordos, A.C.Long, Uncertainty in the manufacturing of fibrous

thermosetting composites: A review, Composites Part A: Applied Science and Manufacturing,

pÿVolume 57, February 2014, Pages 6775

https://dspace.lib.cranfield.ac.uk/handle/1826/9447

Downloaded from Cranfield Library Services E-Repository 